\title{
ANÁLISIS COMPARATIVO DE DOS MÉTODOS DE INVERSIÓN EN TOMOGRAFÍA SÍSMICA EN CROSS-HOLE
}

\begin{abstract}
Resumen
Existen numerosos métodos de inversión por la técnica de tomografía en tiempo de viaje. En este trabajo se aplicaron a datos simulados, dos procedimientos recientemente desarrollados por los autores para la determinación de anomalías convexas, homogéneas e isotrópicas en un medio con las mismas dos últimas características, lo cual permite simplificar la teoría de propagación de ondas considerando rayos rectos. Los emisores y receptores ubicados según el arreglo Cross-Hole y la inclusión ubicada en forma arbitraria en un dominio bidimensional. En el primer método denominado de la Dispersión Mínima (DM) se hizo uso de un criterio de mínima dispersión que condujo a un sistema lineal de ecuaciones. En el segundo, denominado Inversión Variacional (IV) se aplicó el cálculo de variaciones para determinar la forma de la inclusión. Los resultados mostraron la validez de los métodos en lo que respecta a la ubicación, forma y tamaño de la anomalía, aun en presencia de fuertes niveles de ruido en los datos. Finalmente se confrontaron resultados entre las dos técnicas a fin de establecer niveles de eficiencia y robustez entre ellos. Se demostró que el método DM produce en general resultados más confiables que el IV, además de ser más eficiente y no necesitar de tanta información adicional de partida.
\end{abstract}

Palabras claves: tomografía, inversión sísmica, dispersión, cálculo variacional, sistemas lineales.

\begin{abstract}
Several inversion methods can be used to solve travel time tomography problems. In this work two procedures recently developed for the determination of convex, homogeneous and isotropic anomalies in medium with the same two last properties, were applied to simulated data. Homogeneity and isotropy allows simplifying wave propagation theory considering straight rays. Sources and receivers were located according to cross-hole array; and the inclusion positioned in random place in a two-dimensional domain.

On the first method (Dispersion Method, DM), a minimal dispersion criteria was employed that led to a linear system of equations. For the second one (Variational Method, IV), the calculus of variations was used to determine the inclusion's form and location. The results evidenced the validity of the methods considered, relative to the position, form and size of the anomaly, still working with data containing high noise levels. Finally, results were confronted among the two techniques in order to establish levels of efficiency and robustness between them. It was demonstrated that DM leads in general to more reliable results than Variational Method; in addition to be more efficient and need less additional information prior to the inversion process.
\end{abstract}

Keywords: tomography, seismic inversion, dispersion, variational calculus, linear system.

Recibido: 24 de setiembre del 2009 • Aprobado: 12 de abril del 2010.

\section{INTRODUCCIÓN}

Lo e Inderwiesen (1994) definen a la tomografía como "una técnica de inversión que genera una representación gráfica seccional (e.g. un tomograma) de un objeto utilizando la respuesta de este a la energía no destructiva de una fuente externa".
En este trabajo se utilizará la definición anterior a fin de localizar objetos situados en un espacio (dominio), utilizando para ello tiempos de viaje creados artificialmente a partir de un modelo teórico. Se utilizarán, por ende, modelos paramétricos.

Sin embargo se debe destacar que la definición propuesta por Lo e Inderwiesen 
(1994), ha sido ampliada en los últimos años, debido a que la tomografía sísmica en tiempo de viaje permite, además, mapear variaciones en ciertos parámetros en el dominio de estudio; por ejemplo variaciones de compactación del terreno, localización de zonas de humedad, fisuras, etc. Para este caso se utilizan modelos que dividen el dominio en pixeles (Tarantola, 1987; Santamarina \& Cesare, 1994).

Es decir que en definitiva en un sector del terreno o muestra por estudiar, que se denominará como dominio de investigación, se colocan los generadores de ondas sísmicas y en otra ubicación, los receptores de señales, teniendo cuidado de que el contenido de información sea lo más uniforme posible de tal modo que se cubra lo mejor posible el dominio (Santamarina \& Fratta, 2005; Imhof \& Calvo, 2010).

Cuando tanto los emisores como los receptores se ubican en forma vertical, siendo el dominio de trabajo rectangular, el sistema se denomina Cross-Hole. Entre los diversos parámetros físicos del terreno uno de los más significativos en el ámbito de las aplicaciones geotécnicas es la velocidad de transmisión de las ondas de compresión a través del mismo. $\mathrm{Su}$ importancia en relación con los estudios geotécnicos radica en que los valores de este parámetro dependen del grado de compactación e integridad de los materiales $\mathrm{y}$, por lo tanto, están directamente relacionados con la calidad del material estudiado.

En este trabajo, mediante el método de tomografía sísmica por tiempo de viaje se pretende determinar la forma y ubicación de una inclusión (anomalía) inserta en un medio que la contiene. Se considera medio homogéneo e isótropo, por lo cual los rayos que se propagan son rectos. Aunque la presencia de inclusiones produce refracciones (lo que altera la hipótesis de homogeneidad), el error cometido no es grande (Santamarina \& Reed, 1994). Se deberá tener en cuenta, sin embargo, que a mayor contraste de velocidades entre el medio base $\left(V_{I}\right)$ y la inclusión $\left(V_{2}\right)$, mayor será la diferencia en tamaño entre la superficie real y la modelada por cualquier método de inversión (Santamarina, Klein \& Fam, 2002).
La alteración del tiempo de viaje (e.g. primeros arribos) de los rayos, es un indicador de la presencia de una anomalía. Los planteos generales de solución abordan la discretización del dominio en pequeñas celdas rectangulares denominadas pixeles (Imhof, 2007) planteando las ecuaciones de tiempo de viaje recorridos por los rayos, siendo las incógnitas la velocidad (o lentitud) en cada pixel. Se llega a un sistema de ecuaciones lineales:

$$
A \cdot x=y
$$

Donde:

es la matriz de distancias

$x \quad$ el vector de lentitudes (inverso de la velocidad)

$y$ es el vector de tiempos medidos experimentalmente, que es la incógnita buscada

Se trata entonces de un tipo de problema inverso. Planteado el problema desde el punto de vista de división del dominio en pixeles, el sistema formado será generalmente subdeterminado (más incógnitas que ecuaciones), por lo que requerirá incorporar información adicional para su solución. A partir de aquí los métodos difieren, sin existir uno considerado superior. Algunos son: MLS (minimum length solution), DLSS (damped least-squares solution), RLSS (regularized least-squares solution), SVD (descomposición en valores singulares), etc. (Santamarina \& Fratta, 2005). En trabajos anteriores (Imhof \& Calvo, 2003; Calvo \& Imhof, 2005) se intentó un enfoque nuevo utilizando un principio variacional y logrando buenos resultados. Posteriormente se establecieron los fundamentos teóricos del método de la dispersión (Calvo \& Imhof, 2007a; Calvo \& Imhof, 2007b). En esta investigación, luego de presentar una sinopsis de ambas metodologías, se llevará a cabo un análisis comparativo de ambas utilizando datos simulados sin y con presencia de ruidos en dichos datos, a fin de analizar la convergencia de cada uno de los métodos. 


\section{TEORÍA}

Dado un medio homogéneo con velocidad de propagación $V_{1}$, con una inclusión del mismo tipo en su interior con velocidad $V_{2}$; se pretende modelar (determinar su forma y ubicarla) mediante la técnica tomográfica por tiempo de viaje (Sheriff \& Geldard, 1995). Los tiempos de primeros arribos de los rayos originados en los emisores $s_{i}$ y captados en los receptores $r_{j}$, son alterados por la presencia de la inclusión. Interpretar esta alteración permitirá modelar la misma (Figura 1).

Los emisores y receptores se ubican según el dispositivo cross-hole en una región plana; tratándose, por ende, de un problema bidimensional. Tanto el medio base como la inclusión son homogéneos lo que supone propagación de rayos rectos con velocidades $V_{l}$ y $V_{2}$ respectivamente. Además la última es de forma convexa, pudiéndose sustituir por la menos restrictiva condición que un rayo corte a lo sumo en dos puntos al contorno de la misma, excepto en el punto de tangencia.

Como primera medida, a fin de definir la forma de la anomalía se debe determinar cuáles rayos la atraviesan, así como ubicar las coordenadas de los puntos de ingreso $E$ y salida $S$ de los mismos.

Como información del problema se conocen la ubicación de los emisores s y receptores $r$ y los tiempos de viaje entre ellos. También debe ser dato (aún aproximado) la velocidad en la inclusión $V_{2}$.

El valor de $V_{I}$ (para ambos métodos) se calcula de acuerdo con la expresión de la mediana:

$$
V_{1}=\operatorname{median}(\mathbf{V a p})
$$

donde:

Vap $=\left[\frac{D i j}{T i j}\right] i$-ésimo emisor $; j$-ésimo receptor

donde

Vap es el vector formado por el cociente entre las distancias emisor $i$ - receptor $j(D i j), y$ sus tiempos de viaje Tij respectivos.

En otras palabras, para cada par emisor $s_{i}$ receptor $r_{j}$ corresponde un rayo $i-j$ con tiempo de viaje $T i j$ y distancia total recorrida $D i j$; mientras que en el interior de la anomalía la distancia de viaje será di (Figura 2a).

La limitación actual de los dos métodos radica en que $V_{2}$ debe ser dato (additional guess) del problema.

Cada uno de estos escalares que conforman respectivas matrices $\mathbf{T}$ y $\mathbf{D}$, permiten obtener el vector d de distancias recorridas dentro de la anomalía:

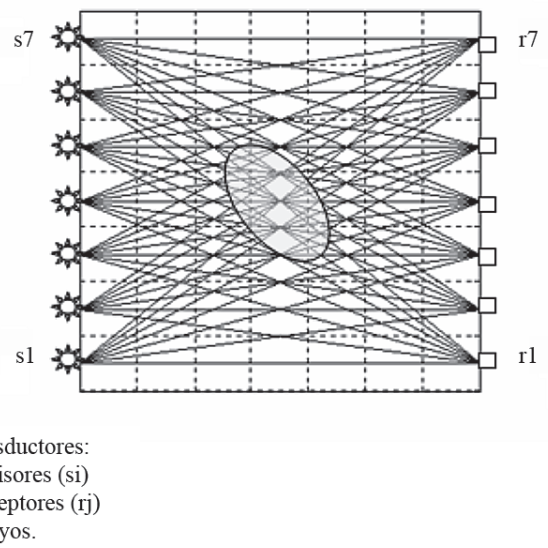

Figura 1. Emisores si, receptores rj. Inclusión de forma elíptica. Configuración Cross-Hole.

Fuente: (Los autores, 2009). 


$$
\mathbf{d}=\left(\mathbf{T}-\frac{\mathbf{D}}{V_{1}}\right)\left(\frac{V_{1} V_{2}}{V_{1}-V_{2}}\right)
$$

Se seleccionan los elementos no nulos de d, renombrando a estos rayos con $k(k=1 . . N)$ y denominando $(x k, y k)$ a la coordenada de $P_{k}$, punto medio de dicho segmento de longitud $d_{k}$. (Figura 2b). Como primera medida se determina el punto central de la anomalía por medios gráficos, según se describe en Imhof y Calvo (2003) para el método variacional; y en forma analítica para el de mínima dispersión, según se detalla en la sección 2.2.

La ubicación de los segmentos (o los puntos $E$ y $S$ ) queda unívocamente determinada por los $x_{k}$, (los $y_{k}$ dependen de estas abscisas). Entonces, la forma y ubicación de la anomalía se resuelve encontrando solo estos valores.

Para la determinación de dichos puntos se desarrollaron dos métodos: a) Inversión Variacional (IV): se determina el mínimo de la funcional longitud del contorno de la anomalía (Imhof \& Calvo, 2003; Calvo \& Imhof, 2005), por lo que se trata de un método iterativo que requiere valores iniciales de $x_{k}$.

b) Dispersión Mínima (DM): Se calcula el mínimo de la función dispersión a partir de la resolución de un sistema lineal de ecuaciones por el método de mínimos cuadrados.

\subsection{Formulación del problema inverso por el Método Variacional}

Sea $y=y(x)$ la ecuación que describe el contorno cerrado C (Figura 3). Denótese como ' $L$ ' a la longitud (perímetro) del mismo; entonces se tendrá una funcional $L=L(y)$ condicionada por 'd', ecuación (2).

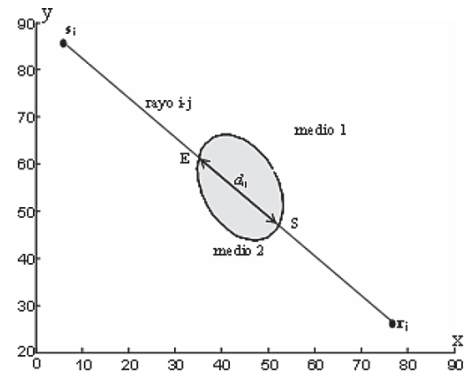

(a)

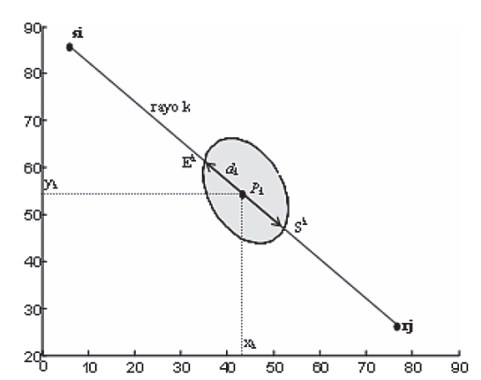

(b)

Figura 2. (a) Trayectoria de un rayo. Puntos E y S de ingreso/egreso del mismo por la inclusión. (b) El rayo $k$ determina $d_{k}$ dentro de la anomalía. $P_{k}$ punto medio del segmento $d_{k}$.

Fuente: (Los autores, 2009).

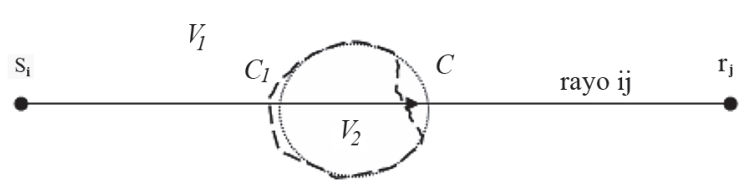

Figura 3. Modificación de la longitud del contorno de la anomalía sujeto a variación $\delta y$.

Fuente: (Los autores, 2009). 
Si a partir del contorno real $C$ se altera $y$ a $y+\delta$ (contorno $C$ ') se notará que $L$ tiende a aumentar; esto es equivalente a sostener que el contorno $C$ corresponde a una solución de longitud mínima (siempre condicionada por $\mathbf{d}$ ).

Se puede aceptar entonces, como una hipótesis plausible (variacional), que el contorno solución corresponde a aquel de longitud mínima:

solución $y / \min L(y) \quad$ (condicionada por $\mathbf{d}$ )

Por lo general no es posible obtener la solución analítica a este problema variacional; por consiguiente se lo plantea por métodos numéricos a partir de una discretización de la curva $y(x)$ reemplazándola por un número finito de puntos.

Es decir que para la inclusión se reemplaza la curva continua por una discretizada conformada por los puntos $P_{i j}^{<i n>}$ y $P_{i j}^{<o u t>}$ (ver más abajo), dando por resultado una poligonal de longitud $\mathrm{L}^{*}$ de fácil determinación.

Debido al hecho de que la matriz de distancias d es conocida, $P_{i j}{ }^{\text {<ou» }}$ es fácilmente calculado a partir de $P_{i j}^{<i n>}\left(x_{i j}^{<i n>} ; y_{i j}^{<i n>}\right)$ y a partir de que se conoce la trayectoria del rayo ij-ésimo; solo $x_{i j}{ }^{<i n>}$ permanece como variable independiente (Figura 4)
Finalmente queda formulado el problema de minimizar la función $\mathrm{L}^{*}$ :

$$
\mathrm{L} *=\mathrm{L} *\left(\mathbf{x}^{<\mathrm{in}>}\right)
$$

Donde

$\mathbf{x}^{<i n>}$

es un vector cuyas $p$ componentes corresponden a los rayos que intersecan la inclusión.

De los métodos de optimización disponibles para encontrar el contorno $\mathrm{C}$ que minimiza el funcional $L(y)$ (sujeto a la restricción d), se optó por el de Nelder-Mead (Strang, 1986) debido a que si bien es más lento que otros, su convergencia es no obstante, asegurada. Además no necesita aplicar derivadas.

\subsection{Formulación por el Principio de la Dispersión Mínima}

Dado el conjunto $P_{k}$; una medida de la dispersión de los mismos se presenta en la ecuación (4) (Montgomery \& Runger, 2004).

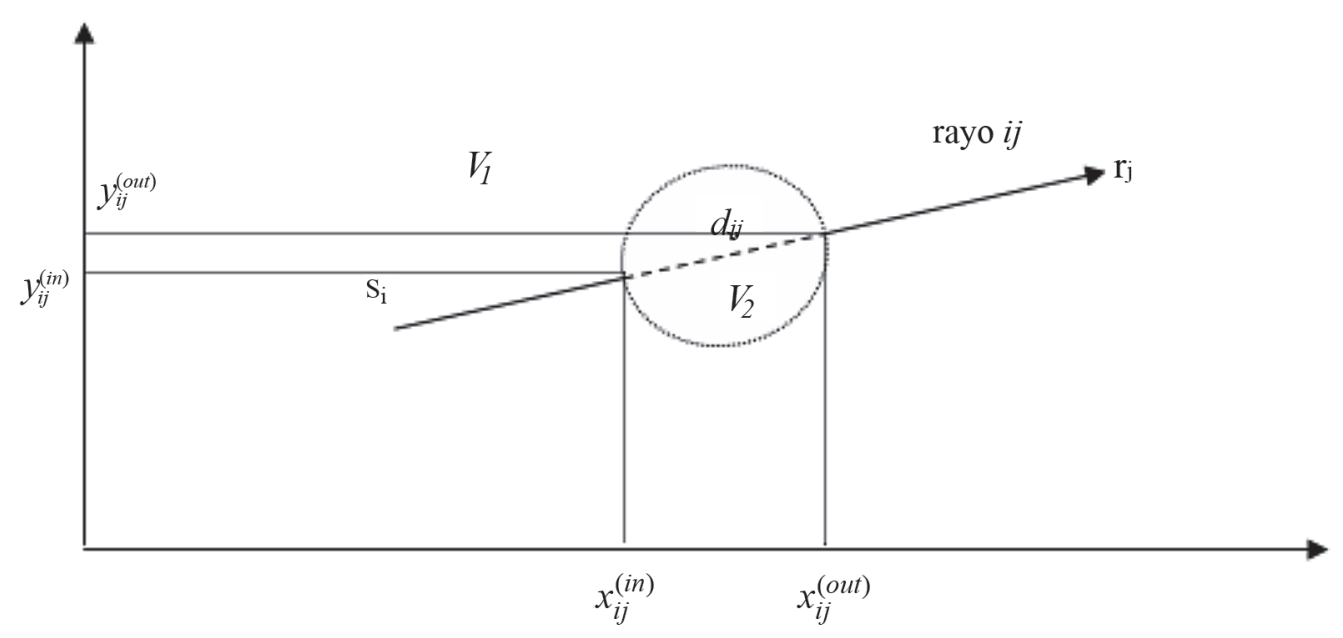

Figura 4. Coordenadas $x i j<i n>$, $x i j<o u t>$ yij<in> y $y i j<o u t>$. Dependen las demás de $x i j<i n>$.

Fuente: (Los autores, 2009). 


$$
\sigma^{2}=\frac{1}{N-1} \sum_{k=1}^{N} w_{k}\left(x_{k}-\bar{x}\right)^{2}+\frac{1}{N-1} \sum_{k=1}^{N} w_{k}\left(y_{k}-\bar{y}\right)^{2}
$$

Donde $\sigma$ es la desviación estándar

$$
\begin{aligned}
& w_{k}=\frac{d_{k}}{L} \\
& L=\sum_{k=1}^{N} d_{k} \\
& \bar{x}=\sum_{j=1}^{N} w_{j} x_{j} \quad \bar{y}=\sum_{j=1}^{N} w_{j} y_{j}
\end{aligned}
$$

La ecuación (5) define los pesos; la ecuación (6) es la longitud total de los rayos en el interior de la inclusión y la ecuación (7) corresponde a las coordenadas del centro de gravedad. Puesto que se conoce la trayectoria recta del rayo $k\left(y=m_{k} x+b_{k} ; \sigma^{2}\right)$; resulta ser solo función de $x_{k}: \sigma^{2}=\sigma^{2}\left(x_{1}, x_{2}, \ldots . x_{n}\right)$.

Los puntos medios de los segmentos $d_{k}$ (es decir los $P_{k}$ ) estarán más cercanos entre sí (e.g. menos dispersos) o no, dependiendo de la ubicación relativa de los segmentos de rayos que intersecan la anomalía. Pero habrá una combinación mutua de los mismos que produzca mínima dispersión. Además, distintas formas de la inclusión, a los que corresponden diferentes valores de la dispersión, se generan al deslizar los segmentos sobre los rayos $k$. En la Figura 5 a-d se observa que al alejarse de la posición óptima, la dispersión de los $P_{k}$ aumenta. Por ello se adopta el siguiente criterio: La forma de la anomalía corresponde a la dispersión mínima de los puntos $P_{k}$.

Las ecuaciones $\frac{\partial \sigma^{2}}{\partial x_{k}}=0$ conducen a la obtención de los $x_{k}$. Se llega a un sistema de ecuaciones de la forma de la ecuación (1), según se verá.

De las ecuaciones (5) a (7) se puede verificar que:

$$
\begin{array}{ll}
\frac{\partial x_{k}}{\partial x_{i}}=\frac{\partial y_{k}}{\partial y_{i}}=\delta_{k i} & \frac{\partial x}{\partial x_{i}}=\frac{\partial y}{\partial y_{i}}=w_{i} \\
y_{i}=m_{i} x_{i}+b_{i} & \frac{\partial y_{i}}{\partial x_{i}}=m_{i}
\end{array}
$$

con $\delta_{k i}$ : delta de Kronecker. Derivando la ecuación (4), reemplazando allí las expresiones (8) y operando (el desarrollo completo se describe en Calvo \& Imhof, 2007) (ver ecuación 9 ).

denominando:

$$
\begin{aligned}
& c_{i j}=\sum_{k=1}^{N}\left(\delta_{k j}-w_{j}\right) w_{k}\left(\delta_{k i}-w_{i}\right) \\
& a_{i j}=c_{i j}\left(1+m_{i} m_{j}\right) \\
& f_{i}=-m_{i} \sum_{j=1}^{N} c_{i j} b_{j}
\end{aligned}
$$

se obtiene finalmente el sistema lineal $(N \times N)$ representado por la ecuación (10) que constituye la forma indicial de la ecuación (4).

$$
a_{i j} x_{j}=f_{i}
$$

La resolución del sistema lineal (10) permite conocer los $x_{k} \mathrm{y}$ de esta forma las coordenadas de los puntos de ingreso $E^{k}$ y salida $S^{k}$ del rayo $k$

$$
\begin{array}{cc}
x_{E}=x_{k}-\frac{d_{k}}{2} \cos \left(\varphi_{k}\right) & x_{S}=x_{k}+\frac{d_{k}}{2} \cos \left(\varphi_{k}\right) \\
y_{E}=y_{k}-\frac{d_{k}}{2} \operatorname{sen}\left(\varphi_{k}\right) & y_{S}=y_{k}+\frac{d_{k}}{2} \operatorname{sen}\left(\varphi_{k}\right) \\
E^{k}=\left(x_{E}, y_{E}\right) & S^{k}=\left(x_{S}, y_{S}\right) \\
y=m_{k} x+b_{k} & \varphi_{k}=\operatorname{arctag}\left(m_{k}\right)
\end{array}
$$

$\sum_{j=1}^{N}\left(\sum_{k=1}^{N}\left(\delta_{k j}-w_{j}\right) w_{k}\left(\delta_{k i}-w_{i}\right)\right)\left(1+m_{i} m_{j}\right) x_{j}=-m_{i} \sum_{j=1}^{N}\left(\sum_{k=1}^{N}\left(\delta_{k j}-w_{j}\right) w_{k}\left(\delta_{k i}-w_{i}\right)\right) b_{j}$ 


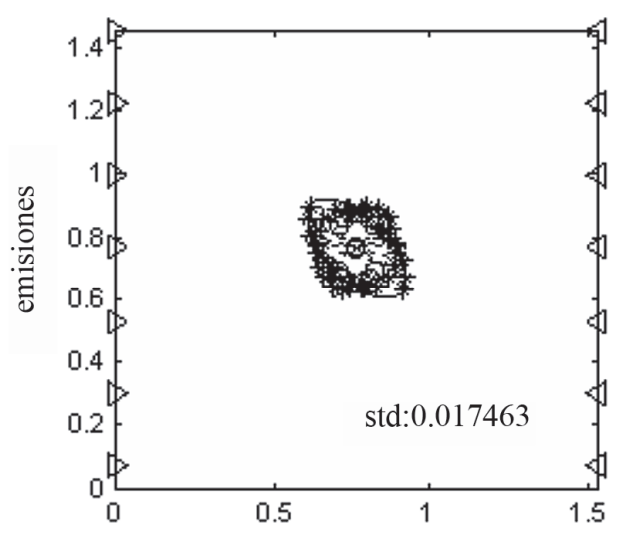

(a)

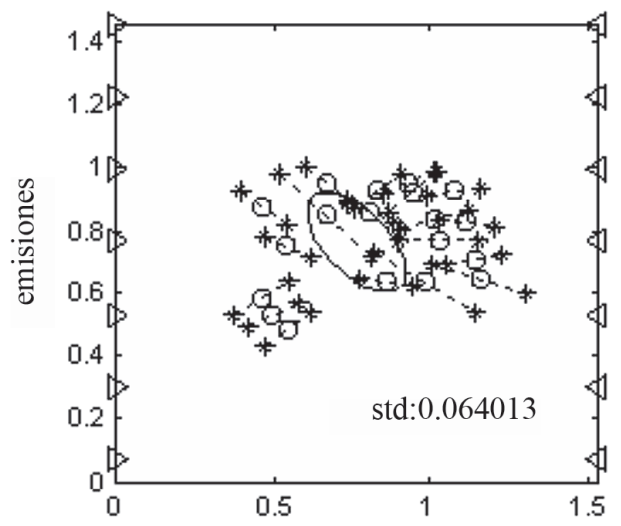

(c)

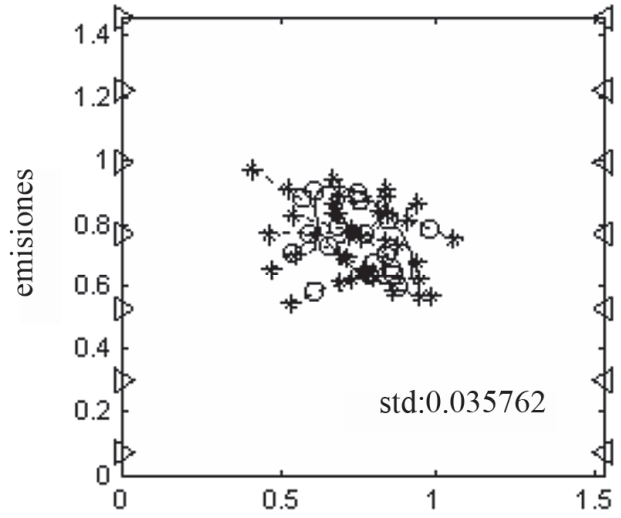

(b)

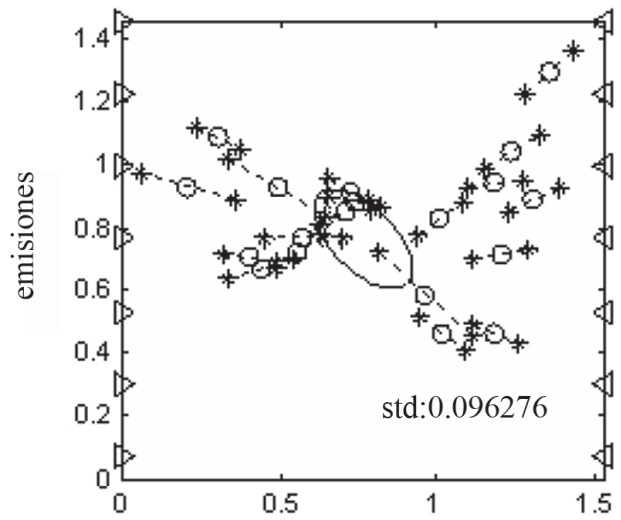

(d)

Figura 5. Método DM. Posiciones relativas de los segmentos para cuatro valores de desviación estándar de los $d k$. Se aprecia con el aumento de la dispersión el alejamiento respecto de la forma real de la inclusión. *: extremo de $d k$; o: $P k$

Fuente: (Los autores, 2009).

Los puntos $E^{k}$ y $S^{k} ; k=1 \ldots . \mathrm{N}$ determinan el contorno aproximado de la anomalía (Figura 6a) También puede ser ajustado por una curva cerrada (e.g. elipse, Figura 6b).

\subsection{Modelos numéricos simulados e inversión de los datos}

Para comparar resultados por ambos métodos se obtuvieron datos de tiempos de viaje predichos a partir de dos modelos teóricos, con la configuración emisor receptor Cross-Hole y ubicación de anomalías de acuerdo con la Figura 7 (Modelos A y B). Tanto el medio base de velocidad $V_{1}$, como la inclusión de $V_{2}$ se consideran como homogéneos e isótropos.

Para la generación de los datos se construyó un algoritmo en plataforma Matlab ${ }^{\circledR}$ que permite a partir del ingreso de las coordenadas de $s i, r i, \mathrm{y}$ datos de la elipse que conforma la inclusión inserta en el medio delimitado por aquellos, junto con las velocidades, predecir los datos de tiempo (primeros arribos) que medirían los ri para un experimento real. El programa permite añadir el nivel de ruido aleatorio deseado, a fin de explorar la convergencia 


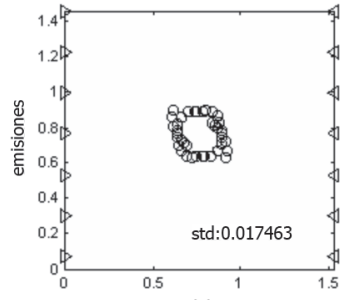

(a)

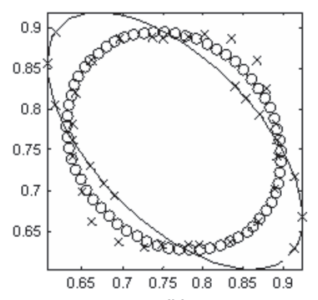

(b)

Figura 6. Resultados de la inversión DM.

(a) forma real de la inclusión y puntos de ajuste con mínima dispersión.

(b) Detalle con ajuste elíptico de los puntos (línea de puntos).

Fuente: (Los autores, 2009).
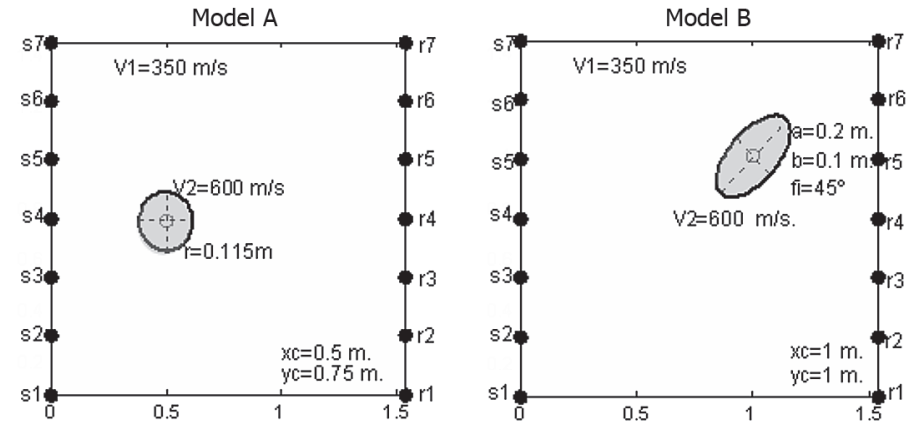

Figura 7. Representación modelos A y B. Cross-Hole. si: emisores; ri: receptores. si-ri $=1.53 \mathrm{~m}$.; s1-s7 = r1-r7 = $1.44 \mathrm{~m}$.

Fuente: (Los autores, 2009).

de los métodos de inversión considerados en situaciones extremas. El desarrollo analítico del algoritmo se describe en el Anexo A.

Se decidió implementar un algoritmo que modelara anomalías elípticas por tratarse la misma de una forma más general que el círculo; ya que permite modelar por ejemplo fracturas, en el caso en que el semieje $a>>b$. Cabe destacar que el círculo es simplemente un caso particular de la elipse en que $a=b$.

Se calcularon datos de tiempo teóricos (predichos) para los dos modelos con varios niveles de ruido, a fin de simular los casos reales de medición en ensayos: nulo $(0 \%) ; 5 \% ; 10 \%$ y hasta $20 \%$. La Figura 8 representa los cuatro niveles de dispersión en los datos, observándose en ordenadas la diferencia entre los tiempos sin anomalía (es decir con solo $V_{l}$ ) y con anomalía $\left(V_{1}\right.$ y $\left.V_{2}\right)$; y en abscisas cada uno de los rayos $(7 s \times 7 r=49$ rayos). Se puede apreciar la dificultad progresiva para identificar los rayos que atraviesan la anomalía.

En la ejecución de los algoritmos se utilizaron los datos de geometría del dispositivo (matriz de 49 $\mathrm{x} 4$ con las coordenadas de emisores y receptores) y tiempos (vectores de $49 \times 1$ ) calculados para Modelos A y B como entrada a los programas de acuerdo con las ecuaciones desarrolladas y que calcularon la inversión por los métodos DM e IV, para los cuatro niveles de error citados en la sección anterior. Posteriormente se analizó la convergencia de los datos y su correlación. 


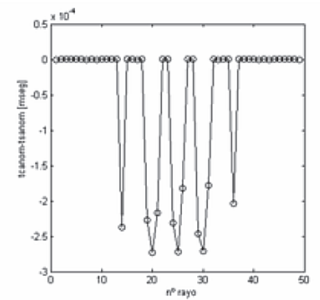

(a)

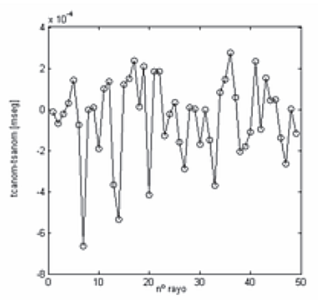

(c)

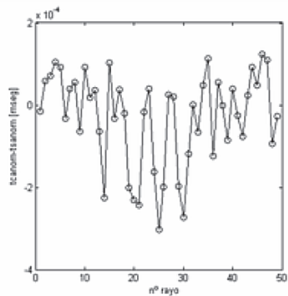

(b)

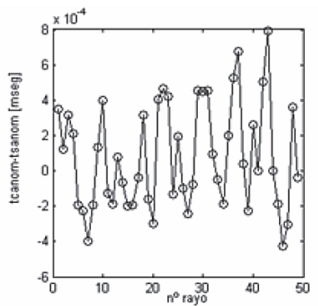

(d)

Figura 8. Diferencias de tiempo en los datos con anomalía para diversos niveles de ruido. (a) $0 \%$; (b) $5 \%$; (c) $10 \%$ y (d) $20 \%$. Ejemplo Modelo B.

Fuente: (Los autores, 2009).

\section{RESULTADOS}

La Figura 9 representa los resultados de la inversión para datos sin ruido, para el DM (Figura 9a y 9b) así como para el IV (Figura 9c y 9d) para ambos modelos A y B descritos.

Se debe tener en cuenta que durante el proceso de inversión con IV se debe inferir (tal como se explicó) el centro de la inclusión por un medio gráfico. Se visualizan todos los rayos que atraviesan la misma y se selecciona el lugar con mayor densidad de rayos. Esto añade un margen de subjetividad que puede actuar en ciertos casos en desmedro al método, cuando no se seleccione en forma adecuada el centro, lo que añade error en forma significativa. Esto no ocurre con el método DM, que lo calcula en forma automática.

Por otra parte, al ser IV un método iterativo, es menos eficiente en cuanto a velocidad. DM solo resuelve un sistema lineal de ecuaciones muy bien condicionado. En cuanto a robustez, los dos funcionan muy bien.

\section{ANÁLISIS COMPARATIVO}

El método DM modela con mayor precisión la forma real de la anomalía. Por ejemplo para el caso de un círculo, IV no reconstruye con total exactitud aquella, sino una elipse (Figuras 9a y 9c, compárese semiejes $a, b$ en ambos casos). Respecto a las coordenadas del centro de la anomalía, prácticamente no existen diferencias.

Para las Figuras 9b y 9d al modelar la elipse, el DM calcula con mayor aproximación la inclinación de la inclusión $\left(37.2^{\circ}\right.$ - IV contra $46^{\circ}$ $\mathrm{DM}$, respecto al real $45^{\circ}$ ).

El resultado anterior es importante, particularmente en exploración geofísica cuando se intenta determinar con la mayor exactitud posible, la ubicación y el buzamiento de estructuras minerales a fin de optimizar costos de perforaciones.

Los Cuadros 1 y 2 (Modelo A) y 2 (Modelo B) presentan los resultados de las inversiones con los dos métodos propuestos para diversos niveles de ruidos. A medida que aumenta el nivel de ruido: 


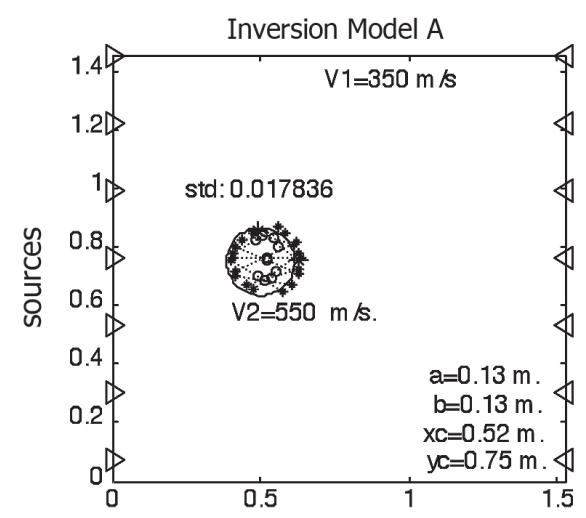

(a)

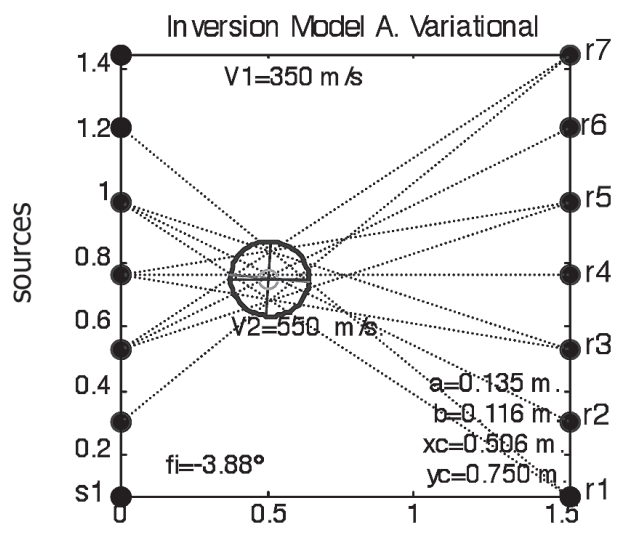

(c)

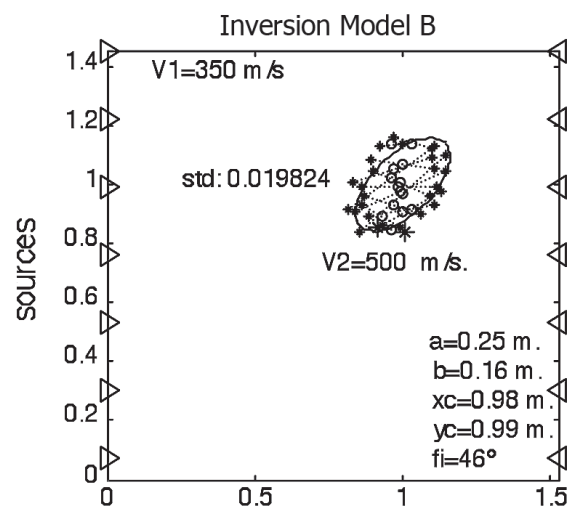

(b)

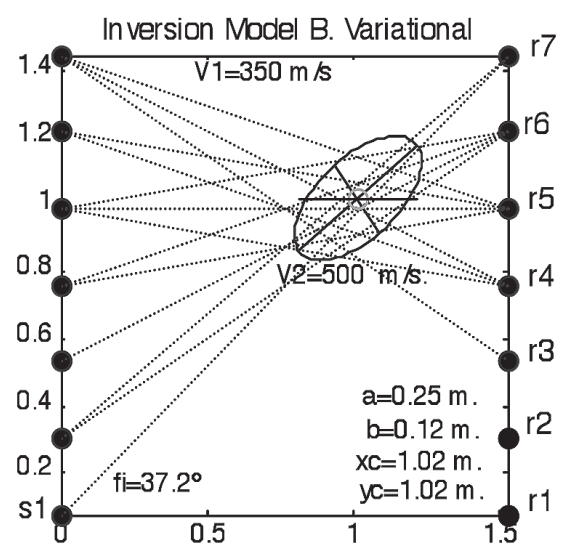

(d)

Figura 9. Resultados de la inversión. Modelos A y B. Sin ruido. (a)-(b): MD. (c)-(d): IV. V1=350 m/s; $\mathrm{V} 2=550 \mathrm{~m} / \mathrm{s}$ (Modelo A); V2=500 m/s (Modelo B)

Fuente: (Los autores, 2009).

a) Disminuye la precisión de los algoritmos para calcular $V_{l}$ (el sistema de cálculo en ambos métodos es idéntico). $V_{2}$ es dato.

b) La capacidad de los dos métodos para localizar el centro de la inclusión $(x c, y c)$ sigue siendo óptima (recuérdese que IV depende del intérprete para esto), aun con márgenes del 20 $\%$ de ruido en los datos.

c) En líneas generales DM presenta leve superioridad sobre IV en la aproximación a los modelos reales de los semiejes $a$ y $b$, así como en el buzamiento de la inclusión. d) A medida que aumenta el ruido en los datos, la forma real de la inclusión se pierde, acercándose progresivamente a un círculo. Los valores de $\phi$ del Cuadro 2 (sombreado) no se deben considerar porque al ser similares $a$ y $b$, la figura tiene aproximadamente aquella forma.

\section{CONCLUSIONES}

1. El método IV requiere la determinación por medio gráfico en pantalla de las coordenadas 
Cuadro 1. Resultados de la inversión de datos. Métodos IV y DM. Modelo A.

\begin{tabular}{|c|c|c|c|c|c|c|c|c|c|}
\hline \multirow{3}{*}{ Parámetro } & \multirow{3}{*}{$\begin{array}{c}\text { Valor } \\
\text { real }\end{array}$} & \multicolumn{8}{|c|}{ Error } \\
\hline & & \multicolumn{2}{|c|}{$0 \%$} & \multicolumn{2}{|c|}{$5 \%$} & \multicolumn{2}{|c|}{$10 \%$} & \multicolumn{2}{|c|}{$20 \%$} \\
\hline & & IV & $\mathrm{DM}$ & IV & $\mathrm{DM}$ & IV & $\mathrm{DM}$ & IV & $\mathrm{DM}$ \\
\hline $\mathrm{xc}(\mathrm{m})$ & 0,5 & 0,51 & 0,52 & 0,51 & 0,48 & 0,37 & 0,45 & 0,76 & 0,76 \\
\hline yc (m) & 0,75 & 0,75 & 0,75 & 0,77 & 0,76 & 0,76 & 0,76 & 0,87 & 0,87 \\
\hline $\mathrm{a}(\mathrm{m})$ & 0,115 & 0,116 & 0,115 & 0,1 & 0,11 & 0,15 & 0,13 & 0,24 & 0,24 \\
\hline $\mathrm{b}(\mathrm{m})$ & 0,115 & 0,113 & 0,115 & 0,11 & 0,11 & 0,08 & 0,1 & 0,13 & 0,13 \\
\hline V1 (m/s) & 350 & 350 & 350 & 341,7 & 341,7 & 337 & 337 & 318,3 & 318,3 \\
\hline $\mathrm{V} 2(\mathrm{~m} / \mathrm{s})$ & 600 & & & & dato $\mathrm{c}$ & lema & & & \\
\hline
\end{tabular}

Fuente: (Los autores, 2009).

Cuadro 2. Resultados de la inversión de datos. Métodos IV y DM. Modelo B.

\begin{tabular}{|c|c|c|c|c|c|c|c|c|c|}
\hline \multirow{3}{*}{ Parámetro } & \multirow{3}{*}{$\begin{array}{c}\text { Valor } \\
\text { real }\end{array}$} & \multicolumn{8}{|c|}{ Error } \\
\hline & & \multicolumn{2}{|c|}{$0 \%$} & \multicolumn{2}{|c|}{$5 \%$} & \multicolumn{2}{|c|}{$10 \%$} & \multicolumn{2}{|c|}{$20 \%$} \\
\hline & & IV & $\mathrm{DM}$ & IV & $\mathrm{DM}$ & IV & $\mathrm{DM}$ & IV & DM \\
\hline $\mathrm{xc}(\mathrm{m})$ & 1 & 1,02 & 0,99 & 1,02 & 0,97 & 1,11 & 1,1 & 1,01 & 1,07 \\
\hline yc (m) & 1 & 0,99 & 1 & 1 & 0,98 & 1,05 & 1,03 & 1,04 & 1,01 \\
\hline $\mathrm{a}(\mathrm{m})$ & 0,2 & 0,19 & 0,18 & 0,18 & 0,17 & 0,24 & 0,23 & 0,17 & 0,16 \\
\hline $\mathrm{b}(\mathrm{m})$ & 0,1 & 0,12 & 0,13 & 0,1 & 0,11 & 0,11 & 0,13 & 0,14 & 0,14 \\
\hline$\phi\left({ }^{\circ}\right)$ & $45^{\circ}$ & 56 & 54 & 37 & 38 & 49 & 50 & -7 & -35 \\
\hline V1 (m/s) & 350 & 350 & 350 & 344 & 344 & 335 & 335 & 334 & 334 \\
\hline $\mathrm{V} 2(\mathrm{~m} / \mathrm{s})$ & 600 & & & & dato & blema & & & \\
\hline
\end{tabular}

Fuente: (Los autores, 2009).

del punto central $x c, y c$ de la anomalía. Por ende, la precisión del método dependerá de la pericia del intérprete en localizarlo. Esto no es necesario con el método MD ya que el algoritmo determina el centro en forma automática a través del cálculo de la dispersión mínima entre el conjunto de los diferentes segmentos $d_{k}$ cuyas longitudes son conocidas. Por otro lado, al no ser un procedimiento iterativo, sino que solo resuelve un sistema lineal de ecuaciones, posee mayor eficiencia computacional.
2. El método IV, si bien es robusto, posee convergencia lenta al utilizar un algoritmo de optimización de Nelder-Mead para la inversión. Por otra parte, la implementación del método DM conduce a un sistema lineal muy bien condicionado y por ende, fácil de resolver por mínimos cuadrados. El procedimiento resulta simple, directo, robusto y de fácil implementación computacional. La experimentación numérica con las distribuciones seleccionadas de emisores, receptores y anomalías para un número alto 
de simulaciones con varios niveles de ruido, demuestra la validez del método en lo que respecta a la ubicación, tamaño y orientación de la anomalía y, en menor medida, referido a la forma.

3. Por último y no menos importante, el método DM permite determinar con mayor precisión el ángulo de buzamiento de las anomalías, lo que resulta muy importante a la hora de calcular costos de perforaciones en aplicaciones de exploración geofísica, por ejemplo, mineras.

\section{RECONOCIMIENTOS}

Este trabajo de investigación forma parte de los proyectos Método de inversión de anomalías en medios heterogéneos y anisotrópicos" y Detección y modelado de inclusiones en arena por tomografía sísmica en Cross Hole, subvencionados por la Secretaría de Ciencia y Técnica (CICITCA) de la Universidad Nacional de San Juan.

\section{REFERENCIAS BIBLIOGRÁFICAS}

Calvo, C. A. \& Imhof, A. L. (2005). A variational formulation to image inclusions. Scientific Session of MEPhI, 5, 156-158.

Calvo, C.A. \& Imhof,A. L. (2007a). Detección de anomalías 2D por un método de inversión por mínima dispersión. Revista Internacional de Métodos Numéricos para Cálculo y Diseño en Ingeniería, 23(3), 295-304.

Calvo, C. A. \& Imhof, A. L. (2007b). Minimum dispersion method for anomaly detection in the plane. Annals of the Moscow Institute of Physics (Mephi).

Imhof, A. L. \& Calvo, C. A. (2003). A variational formulation to image inclusions in 2D Travel Time Tomography. Brazilian Journal of Geophysics, 21(3).

Imhof, A. L. (2007). Caracterización de arenas y gravas con ondas elásticas: tomografía sísmica en cross-hole. Tesis de Doctorado, Facultad de Ingeniería, Universidad Nacional de Cuyo, Mendoza, Argentina.

Imhof, A. L. \& Calvo, C. A. (2010). Seismic data inversion by Cross-Hole tomography using geometrically uniform spatial coverage. Brazilian Journal of Geophysics, 28(1). (en prensa).

Lo, T. W. \& Inderwiesen, P. (1994). Fundamentals of seismic tomography. Geophysical Monograph Series $\left(\mathrm{N}^{\circ} 6\right)$. Ed. Society of Exploration Geophysicists (SEG).

Montgomery, D. C. \& Runger, G. C. (2004). Probabilidad y estadística aplicada a la Ingeniería. México D.F.: McGraw-Hill.

Santamarina, J. C. \& Cesare, M. A. (1994). Velocity inversion in the near surface: vertical heterogeneity and anisotropy. Internal Report.University of Waterloo. Canada.

Santamarina, J. C. \& Fratta, D. (2005). Discrete signals and inverse problems. An introduction for engineers and scientists. UK: Wiley \& Sons.

Santamarina, J. C. \& Reed, A. C. (1994). Ray Tomography: Errors and Error Functions. Journal of Applied Geophysics, 32, 347-355.

Santamarina, J. C., Klein, K. A. \& Fam, M. (2002). Soils and waves. UK: Wiley \& Sons.

Sheriff, R. E. \& Geldard, L. P. (1995). Exploration seismology. $2^{\mathrm{a}}$ ed. New York: Cambridge University Press.

Strang, G. (1986). Álgebra lineal y sus aplicaciones. Addison Wesley Iberoamericana.

Tarantola, A. (1987). Inverse problem theory. Amsterdam: Elsevier. 613 p. 


\section{SOBRE LOS AUTORES}

\section{Armando Luis Imhof}

Geofísico-BSc. en Geofísica. Especialista en Gestión Ambiental. Doctor en Ingeniería Instituto Geofísico Sismológico Volponi Facultad de Ciencias Exactas, Físicas y Naturales Universidad Nacional de San Juan, Argentina.
Correo electrónico: aimhof@unsj.edu.ar

\section{Carlos Adolfo Calvo}

Ingeniero Electromecánico. Magister en Matemática Aplicada. Depto de Matemática Aplicada. Facultad de Ingeniería.

Universidad Nacional de San Juan, Argentina. Correo electrónico: ccalvo@unsj.edu.ar 


\section{ANEXO A}

\section{Desarrollo analítico del algoritmo para generar datos}

El algoritmo modela una elipse rotada que por otra parte es la cónica que mejor ajusta la forma de un cuerpo irregular. Los pasos lógicos para realizar el programa son:

a. Generar matrices con las coordenadas $x$, y de los emisores y receptores (cualquier número). Calcular los tiempos de arribo (asunción de rayo recto, medio homogéneo e isótropo) sin considerar presencia de anomalías. Considerar la velocidad del medio base $V_{l}$.

b. Representar una elipse (inclusión, $V_{2}$ ) inserta por completo en el dominio (medio base).

c. Interceptar la elipse con los rayos emisor-receptor que la atraviesan y determinar la longitud recorrida en el interior de la misma por cada rayo y su contribución al tiempo global (e.g. el tiempo medido de primer arribo en un caso real).

d. Calcular con las diferencias de tiempos encontradas, el tiempo medido predicho (teórico)

La expresión general de una elipse en cualquier sistema de coordenadas es:

$$
\frac{X^{2}}{a^{2}}+\frac{Y^{2}}{b^{2}}=1
$$

Por otra parte, las ecuaciones que expresan el sistema rotado y trasladado $(X, Y)$ en función del de referencia $(x, y)$ es:

$$
\left\{\begin{array}{c}
X \\
Y
\end{array}\right\}=\left[\begin{array}{cc}
\cos \varphi & \operatorname{sen} \varphi \\
-\operatorname{sen} \varphi & \cos \varphi
\end{array}\right] \cdot\left\{\begin{array}{c}
(x-x c) \\
y-y c
\end{array}\right\}
$$

Reemplazando $X, Y$ de la ecuación (A.2) en la expresión (A.1) y operando, se obtendrá la siguiente ecuación que representa la elipse en un sistema rotado y trasladado $(x c, y c)$ del origen:

donde:

$$
\begin{gathered}
A x^{2}+B x y+C y^{2}+D x+E y+F=0 \\
A=\left(\frac{c}{a}\right)^{2}+\left(\frac{s}{b}\right)^{2} \\
B=2 c s\left(\frac{1}{a^{2}}-\frac{1}{b^{2}}\right) \\
C=\left(\frac{s}{a}\right)^{2}+\left(\frac{c}{b}\right)^{2} \\
D=-\frac{2 x c \cdot c^{2}}{a^{2}}-\frac{2 x c \cdot s^{2}}{b^{2}}-\frac{2 y c \cdot c \cdot s}{a^{2}}+\frac{2 y c \cdot c \cdot s}{b^{2}}
\end{gathered}
$$


(...continuación de la fórmula A.4)

$$
\begin{aligned}
& D=-\frac{2 x c \cdot c^{2}}{a^{2}}-\frac{2 x c \cdot s^{2}}{b^{2}}-\frac{2 y c \cdot c \cdot s}{a^{2}}+\frac{2 y c \cdot c \cdot s}{b^{2}} \\
& E=-\frac{2 y c \cdot s^{2}}{a^{2}}-\frac{2 y c \cdot c^{2}}{b^{2}}-\frac{2 x c \cdot c \cdot s}{a^{2}}+\frac{2 x c \cdot c \cdot s}{b^{2}} \\
& F=\left(\frac{x c \cdot c}{a}\right)^{2}+\left(\frac{y c \cdot s}{a}\right)^{2}+\left(\frac{x c \cdot s}{b}\right)^{2}+\left(\frac{y c \cdot c}{b}\right)^{2}+\frac{2 x c \cdot y c \cdot s \cdot c}{a^{2}}-\frac{2 x c \cdot y c \cdot s \cdot c}{b^{2}}-1 \\
& c=\cos \varphi \\
& s=\sin \varphi
\end{aligned}
$$

A continuación se intercepta la recta (rayo) con la elipse:

$$
\left\{\begin{array}{l}
y=m x+b b \\
A x^{2}+B x y+C y^{2}+D x+E y+F=0
\end{array}\right.
$$

Este constituye un sistema en $x$ al que se le calculan las raíces $x 1$ y $x 2$, que serán las abcisas de los puntos de intersección:

$$
\text { raices } x 1, x 2 \Rightarrow\left\{\begin{array}{lr}
\text { reales } & y 1, y 2=m(x 1, x 2)+b b \\
\text { imaginarias } & \text { no existe } \bigcap .
\end{array}\right.
$$

Se reemplazó el valor de $y$ de la ecuación A.5 (a) en la (b), obteniéndose una ecuación polinómica en $\mathrm{x}$ de orden 2:

$A 0 \mathrm{x}^{2}+A 1 x+A 2=0$

$$
A 0 x^{2}+A 1 x+A 2=0
$$

donde:

$$
\begin{aligned}
& A 0=A+B \cdot m+C \cdot m^{2} \\
& A 1=B \cdot b b+2 \cdot C \cdot m \cdot b b+D+E \cdot m \\
& A 2=C \cdot b b^{2}+E \cdot b b+F
\end{aligned}
$$

El comando matlab roots ([A0 A1 A2]) calcula las raíces $(x 1, x 2)$; luego reemplazando en la ecuación A.5 (a) se obtendrá el par $(y 1, y 2)$.

Finalmente, aplicando el Teorema de Pitágoras se calculan las distancias $d$ en el interior de la anomalía para todos los rayos y el tiempo perturbado debido a los mismos. Finalmente, se representa gráficamente. 
